\title{
A nonextensive statistics approach for Langmuir waves in relativistic plasmas
}

\author{
V. Muñoz ${ }^{1,2}$ \\ ${ }^{1}$ Departamento de Física, Facultad de Ciencias, Universidad de Chile, Casilla 653, Santiago, Chile \\ ${ }^{2}$ Department of Earth System Science and Technology, Kyushu University, Fukuoka 816-8580, Japan
}

Received: 16 January 2006 - Revised: 8 May 2006 - Accepted: 17 May 2006 - Published: 29 June 2006

\begin{abstract}
The nonextensive statistics formalism proposed by Tsallis has found many applications in systems with memory effects, long range spatial correlations, and in general whenever the phase space has fractal or multi-fractal structure. These features may appear naturally in turbulent or non-neutral plasmas. In fact, the equilibrium distribution functions which maximize the nonextensive entropy strongly resemble the non-Maxwellian particle distribution functions observed in space and laboratory and turbulent pure electron plasmas.

In this article we apply the Tsallis entropy formalism to the problem of longitudinal oscillations in a proton-electron plasma. In particular, we study the equilibrium distribution function and the dispersion relation of longitudinal oscillations in a relativistic plasma, finding interesting differences with the nonrelativistic treatment.
\end{abstract}

\section{Introduction}

Traditionally, the equilibrium of statistical systems has been studied based on the Boltzmann-Gibbs entropy,

$S_{\mathrm{BG}}=-k_{B} \sum_{i} p_{i} \ln p_{i}$,

where $k_{B}$ is a constant, and $p_{i}$ is the probability of the $i$-th microstate. Now let us consider two independent systems $A$, $B$, that is, the probability of the system $A+B$ of being in a state $i+j$, where $i$ is a microstate of $A$ and $j$ a microstate of $B$, is:

$p_{i+j}^{A+B}=p_{i}^{A} \cdot p_{j}^{B}$.

In this case, the Boltzmann-Gibbs entropy satisfies

$S_{\mathrm{BG}}^{A+B}=S_{B G}^{A}+S_{B G}^{B}$,

Correspondence to: V. Muñoz

(vmunoz@macul.ciencias.uchile.cl) that is, the entropy is an extensive quantity. The statistical description based on this entropy has been successfully applied to a wide variety of systems for over a century.

However, an increasing amount of experimental, computational and theoretical evidence, shows that this formalism is not always adequate. An interesting example is anomalous diffusion. In standard statistical mechanics, diffusive processes involve walks where each step is independent of the previous ones. This leads to diffusion rates proportional to $\sqrt{t}$, where $t$ is the time, whereas anomalous diffusion is either faster or slower. This does not fit the standard framework. A closely related fact is that in Boltzmann-Gibbs statistics, equilibrium distribution functions are MaxwellBoltzmann ones. But although such distribution functions are ubiquitous in Nature, they do not describe all possible processes. Distribution of citations of scientific articles or the distribution of time intervals between consecutive heartbeats (Peng et al., 1993), for instance, are better described by power laws or Lévy distribution functions. In general, the Boltzmann-Gibbs formalism fails for systems with long range interactions, memory effects, and, in general, systems where the relevant phase space has fractal or multifractal structure.

In order to address this problem, Tsallis (1988) proposed the following entropy:

$S_{q}=k_{B} \frac{1-\sum_{i} p_{i}^{q}}{q-1}$,

where $q$ is a real number. For $q=1, S_{q}$ reduces to $S_{\mathrm{BG}}$, and therefore can be regarded as a generalization of the Boltzmann-Gibbs entropy.

$S_{q}$ has the basic property that for independent systems $A$ and $B$, in the same sense as defined above, the entropy of the composite system $A+B$ is given by:

$S_{q}(A+B)=S_{q}(A)+S_{q}(B)+(1-q) S_{q}(A) S_{q}(B) / k_{B}$.

Published by Copernicus GmbH on behalf of the European Geosciences Union and the American Geophysical Union. 


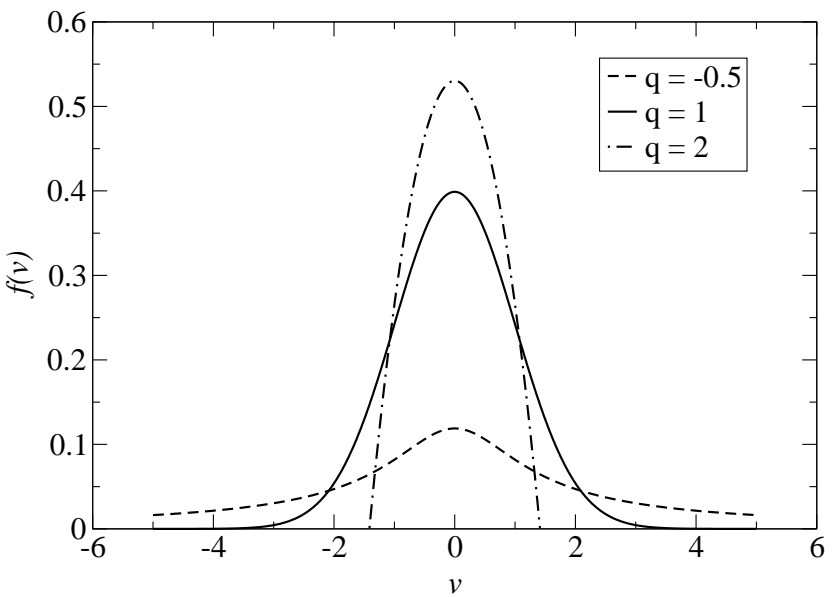

Fig. 1. Velocity distribution function gas of nonrelativistic particles of energy $E=m v^{2} / 2$, for various values of $q$.

Thus, $q$ is a measure of the nonextensivity. Statistical mechanics can be consistently formulated in terms of this generalized entropy. Results have been encouraging. Among the various physical systems where connections with the Tsallis entropy formalism have been found are gravitational systems (Taruya and aki Sakagami, 2003), long range Hamiltonian systems (Latora et al., 2001), nonlinear inverse bremsstrahlung absorption in plasmas (Tsallis and de Souza, 1997), turbulence (Arimitsu and Arimitsu, 2000), and granular systems (Sattin, 2003), and many applications in astrophysics, fluid dynamics, biology, economy, quantum mechanics, and others (Abe and Okamoto, 2001).

Another important property of the nonextensive formalism is that the distribution function which maximises $S_{q}$ is nonMaxwellian (Silva Jr. et al., 1998; Lima et al., 2000). Rather, it is given by an expression like Eq. (5) below. To illustrate it, in Fig. 1 we plot the equilibrium distribution function for a gas of nonrelativistic particles, such that $E=m v^{2} / 2$, for a few values of $q$ :

For $q=1$, the Maxwell distribution function is obtained. But for $q<1$, high energy states are more probable than in the extensive case. The distribution corresponds to a Lévy function, with power law behavior for large energy. On the other hand, for $q>1$ high energy states are less probable than in the extensive case, and there is a cutoff beyond which no states exist. For a nonrelativistic gas in one dimension, $E=m v^{2} / 2$, and the cutoff is (Lima et al., 2000)

$v_{\max }=\sqrt{2 k_{B} T / m(q-1)}$.

This result is important because, as stated above, many systems exhibit non-Gaussianity in their distribution functions. For instance, anomalous diffusion can be described in a natural way by the nonextensive formalism (Prato and Tsallis, 1999), and the same is true for any other system where Lévy flights or distribution functions are relevant.
In particular, velocity distributions in plasmas are often found to be non-Maxwellian. Lévy or Kappa distribution functions are common in space and laboratory plasmas (Mauk et al., 1987; Scudder et al., 1981; Marsch et al., 1982; Collier et al., 1996; Liu et al., 1994). Radial density profiles, with a cutoff in the particle distribution, can be sustained for long times in turbulent electron plasmas (Huang and Driscoll, 1994). As such distribution functions emerge naturally from a nonextensive statistical description, several authors have successfully applied this approach to plasma systems (Boghosian, 1996; Anteneodo and Tsallis, 1997; Tsallis and de Souza, 1997; Leubner, 2004).

Our interest here is to consider wave propagation in plasmas. For a collisionless thermal plasma, a dispersion relation for electrostatic waves can be found and solved for an equilibrium distribution function which maximises Eq. (2) (Lima et al., 2000). The dispersion relation is found to fit experimental data better than if the energy distribution is given by a Maxwellian.

This analysis was restricted to the non-relativistic regime. However, relativistic plasmas are present in many physical systems, like pulsar magnetospheres (Curtis, 1991; Ruderman and Sutherland, 1975; Cheng and Ruderman, 1980), AGN jets (Wardle et al., 1998; Hirotani et al., 2000; Wardle and Homan, 2001) and experiments in laser plasma interactions (Mourou and Umstadter, 1992; Fuchs et al., 1999). On the other hand, non-neutrality and/or turbulence are relevant to several of these systems (Luo et al., 2002; Schlickeiser et al., 2002; Naumova et al., 2001). Thus, it is of interest to study such relativistic systems using a nonextensive statistic approach. In this paper, we intend to generalize the work of Lima et al. (2000) to the relativistic case, based on the relativistic version of the Tsallis formalism (Lavagno, 2002). In particular, we will examine some general properties of the equilibrium distribution function and the frequency for electrostatic waves in the long wavelength limit for ultrarelativistic plasmas.

\section{Relativistic distribution function}

In the nonextensive description, the equilibrium distribution function for a relativistic plasma can be written (Lavagno, 2002)

$f_{q}(\boldsymbol{p})=A_{q}\left[1-(q-1) \frac{E}{k_{B} T}\right]^{1 /(q-1)}$,

where

$E=\sqrt{m^{2} c^{4}+\boldsymbol{p}^{2} c^{2}}$,

is the particle energy and $A_{q}$ is a normalization constant. For $q=1$, the extensive limit is recovered, and Eq. (5) yields the Boltzmann distribution function $f_{q=1}=A_{1} \exp \left(-E / k_{B} T\right)$.

If $q-1<0$, Eq. (5) is real for any value of $E \geq 0$. However, if $q-1>0, f_{q}$ is real only if the quantity in square brackets 
in Eq. (5) is positive. That is,

$$
\begin{array}{cc}
\text { if } q \geq 1 & 0 \leq E \leq \frac{k_{B} T}{q-1}, \\
\text { if } q \leq 1 & 0 \leq E .
\end{array}
$$

If $q \geq 1$ there is an energy cutoff. Both Eqs. (7) and (8) hold for the extensive limit $q=1$, in which case they give the expected constraint $0 \leq E<\infty$.

Since there is no energy cutoff for $q<1$, normalizability of the distribution function is not guaranteed. For a onedimensional plasma, if $\boldsymbol{p}=p \hat{z}$, it turns out that $\int d p f_{q}(p)$ is finite only if $q>0$.

On the other hand, if $q \geq 1$, from the cutoff in Eqs. (7) and (6) it follows that

$|p| \leq \frac{1}{c} \sqrt{\left(\frac{k_{B} T}{(q-1)}\right)^{2}-m^{2} c^{4}}$.

Thus, a real value for the cutoff in Eq. (9) exists only if

$q \leq 1+\frac{k_{B} T}{m c^{2}}$.

Finally, then, unlike the nonrelativistic case (Lima et al., 2000), where $-1 \leq q<\infty$, in the relativistic regime $0<q \leq 1+k_{B} T / m c^{2}$. Two cases arise depending on the sign of $q-1$ :

\section{a) $0<q \leq 1$.}

Energy can take any value

$0 \leq E<\infty$.

b) $1 \leq q \leq 1+\frac{k_{B} T}{m c^{2}}$.

Energy is constrained to

$$
0 \leq E \leq \frac{k_{B} T}{q-1},
$$

i.e.

$$
0 \leq|p| \leq \frac{1}{c} \sqrt{\left(\frac{k_{B} T}{(q-1)}\right)^{2}-m^{2} c^{4}} .
$$

\section{Dispersion relation for longitudinal plasma oscilla- tions}

We consider a proton-electron relativistic plasma. Assuming protons remain at rest, and that particles only move in one dimension $(\hat{z})$, linearization of Poisson and Vlasov equations, and a Fourier transform in space and a Laplace transform in time, yields the following dispersion relation for longitudinal oscillations:

$1=i \frac{\omega_{p}^{2}}{k} m \int d p_{z} \frac{\partial f_{q}\left(p_{z}\right) / \partial p_{z}}{s+i k v_{z}}$, where $\omega_{p}$ is the plasma frequency, $k$ is the wavenumber, $s$ is the argument of the Laplace transform $(s=-i \omega$, where $\omega$ is the wave frequency, if there is no damping), $m$ the electron mass, $p=m \gamma v$ is the particle momentum, and $f_{q}(p, z)$ is the equilibrium electron distribution function, normalized to unity. Particle energy is $E=\sqrt{m^{2} c^{4}+p_{z}^{2} c^{2}}$.

Following Lima et al. (2000), we now consider the long wavelength limit $k \ll k_{D}$, where $k_{D}=2 \pi / \lambda_{D}$, and $\lambda_{D}$ is the Debye length. The integrand has a pole at $v=-i s / k$. If Im $v \ll 1$, integration can be performed on the real axis. Then Eq. (11) can be written, to lowest order in $k$,

$1=-\frac{\omega_{p}^{2}}{s^{2}} \int_{-p_{\max }}^{p_{\max }} d p_{z} \frac{f_{q}\left(p_{z}\right)}{\gamma^{3}}$

where $\gamma=\left(1+p_{z}^{2} / m^{2} c^{2}\right)^{1 / 2} . \quad p_{\max }$ is the maximum momentum allowed by Eq. (9) if $1 \leq q \leq 1+k_{B} T / m c^{2}$, or $\infty$ if $0<q \leq 1$.

Equation (12) depends on $q$ in general, and therefore, unlike the nonrelativistic case (Lima et al., 2000), nonextensive corrections to the dispersion relation of longitudinal waves may appear to the lowest order in $k v_{z} / s$. This will be shown explicitly in Sect. 4 for the ultra-relativistic plasma. Also, as the integrals in Eqs. (11) and (12) are always finite if $f_{q}$ is normalizable, there are no further conditions for $q$.

\section{Ultra-relativistic plasma}

If temperatures are ultra-relativistic, $k_{B} T \gg m c^{2}$. In this case, we can consider $\left|p_{z}\right| \gg m c$, since the contribution of particles not satisfying this condition would be negligible. Then $E \simeq|p| c$, and the integral in Eq. (12) can be done analytically.

As shown in Sect. 2, we must distinguish the cases $q>1$ and $q<1$.

\section{$4.10<q \leq 1$}

Since $p_{\max }=\infty$, in this case, the dispersion relation (12) yields the frequency of longitudinal oscillations

$\omega^{2}=-s^{2}=\omega_{p}^{2} \frac{1}{\tau} q I\left(\tau,-\frac{1}{q-1}\right)$,

where

$\tau=\frac{k_{B} T}{m c^{2}}$, 


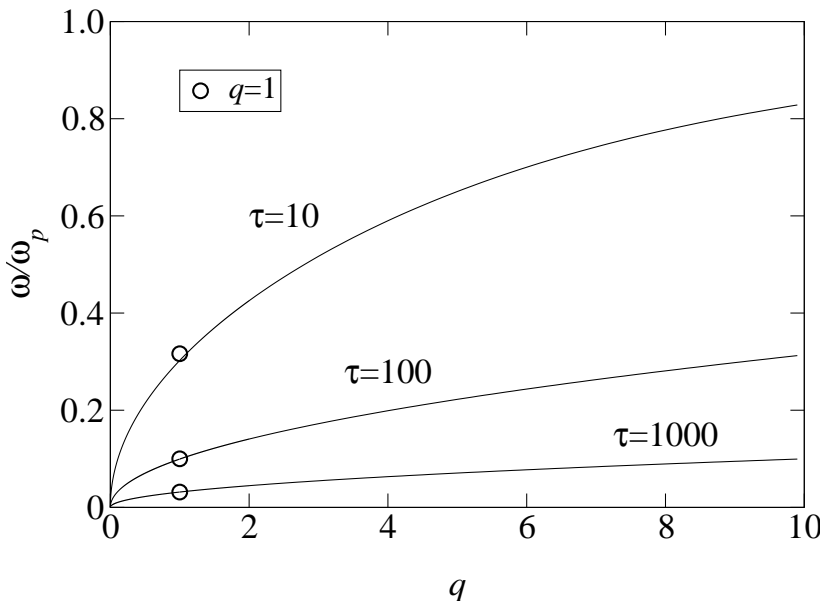

Fig. 2. Frequency $\omega$ vs. $q$, Eqs. (13) and (17).

and

$$
\begin{aligned}
& I(\tau, \alpha)=-\frac{1}{\sqrt{\pi}}(\tau \alpha)^{\alpha+1} \alpha \Gamma\left(\frac{3+\alpha}{2}\right) \Gamma\left(-\frac{\alpha}{2}\right) \\
& \quad \times{ }_{2} F_{1}\left(\frac{1+\alpha}{2}, \frac{3+\alpha}{2}, \frac{3}{2} ;-\alpha^{2} \tau^{2}\right) \\
& +\frac{1}{\sqrt{\pi}}(\tau \alpha)^{\alpha} \Gamma\left(\frac{1-\alpha}{2}\right) \Gamma\left(\frac{2+\alpha}{2}\right) \\
& \quad \times{ }_{2} F_{1}\left(\frac{2+\alpha}{2}, \frac{\alpha}{2}, \frac{1}{2} ;-\alpha^{2} \tau^{2}\right) \\
& +\frac{\tau \alpha}{-1+\alpha}{ }_{3} F_{2}\left[\left\{\frac{1}{2}, 1, \frac{3}{2}\right\},\left\{\frac{2-\alpha}{2}, \frac{3-\alpha}{2}\right\},-\tau^{2} \alpha^{2}\right]
\end{aligned}
$$

Here, ${ }_{2} F_{1}$ is the hypergeometric function, and ${ }_{m} F_{n}$ is the generalized hypergeometric function,

$$
\begin{aligned}
{ }_{m} & F_{n}\left(\left\{a_{1}, \ldots, a_{m}\right\},\left\{b_{1}, \ldots, b_{n}\right\}, z\right) \\
= & \sum_{\nu=0}^{\infty} \frac{\left(a_{1}\right)_{\nu} \cdots\left(a_{m}\right)_{v}}{\left(b_{1}\right)_{v} \cdots\left(b_{n}\right)_{v}} \frac{z^{\nu}}{v !},
\end{aligned}
$$

with $(a)_{v}=a(a+1) \cdots(a+v-1)$.

\section{$4.21 \leq q \leq 1+k_{B} T / m c^{2}$}

Now $p_{\max }$ is given by Eq. (9). The frequency of longitudinal oscillations is:

$\omega^{2}=-s^{2}=\omega_{p}^{2} J\left(\tau, \frac{1}{q-1}\right)$,

where

$J(\tau, \alpha)={ }_{3} F_{2}\left(\left\{\frac{1}{2}, 1, \frac{3}{2}\right\},\left\{1+\frac{\alpha}{2}, \frac{3}{2}, \frac{\alpha}{2}\right\},-\alpha^{2} \tau^{2}\right)$

Using Eqs. (13) and (17), the frequency can be plotted as a function of the nonextensivity parameter $q$. This is shown in Fig. 2, for a few values of $\tau$.
If the usual Boltzmann-Gibbs statistics is used, the plasma frequency for a one-dimensional ultra-relativistic plasma is

$\omega=\frac{\omega_{p}}{\sqrt{\tau}}$.

This result is plotted as circles in Fig. 2. Agreement is very good, confirming that the extensive limit is indeed recovered in our analytical results for $q=1$. However, for low $\tau$ ( $\tau=10$ in Fig. 2), the extensive result is slightly off the corresponding curve. This is also consistent with our approximations. In effect, as shown in Eq. (9), higher values of $q$ imply a very narrow range of possible momenta. Therefore, if $q$ is too large, this will conflict with our assumption $\left|p_{z}\right| \gg m c$ used to obtain Eqs. (13) and (17). As the maximum value of $q$ is given by $q=1+\tau$, this means that the ultra-relativistic approximation discussed here holds if $q \ll \tau$. The extensive limit $q=1$, then, will be better approximated for $\tau \gg 1$, as seen in Fig. 2.

\section{Conclusions}

In this paper, we have considered a relativistic plasma whose statistics is not described by the traditional Boltzmann-Gibbs entropy, but by the generalized Tsallis entropy. Here, a parameter $q$ in the entropy characterizes the degree of nonextensivity in the system.

The equilibrium distribution function is calculated. Mathematical constrains on $f_{q}$ determine that $q$ is restricted to values $0<q \leq 1+\tau$, where $\tau=k_{B} T / m c^{2}$, where $T$ is the plasma temperature and $m$ the particle mass. These bounds are much tighter than the corresponding ones in the nonrelativistic theory $(-1 \leq q<\infty)$, and depend on physical parameters, unlike the nonrelativistic case.

We apply the nonextensive formalism to longitudinal oscillations in the plasma. We obtain a dispersion relation for the long wavelength limit. Nonextensive effects modify the frequency even at zeroth-order in $k$. By contrast, in the nonrelativistic regime, nonextensive corrections only appear when dispersion effects are considered.

Analytic expressions in terms of hypergeometric functions have been obtained for the frequency of oscillations for ultrarelativistic temperatures, so that it can be explicitly calculated. The result reduces to the expected extensive result for $q=1$.

There are two important approximations in the present analysis, namely, infinite wavelength and ultra-relativistic regime. These limitations will be dealt with in future work. In particular, it is of interest to consider finite wavelength, which introduces dispersion and damping effects in the system.

Acknowledgements. V. Muñoz wishes to acknowledge a Postdoctoral Fellowship granted by JSPS (Japan). This work has been partially funded by FONDECYT research grant No. 1060830. 
Edited by: P.-L. Sulem

Reviewed by: A. Lavagno and another referee

\section{References}

Abe, S. and Okamoto, Y.: Nonextensive Statistical Mechanics and its Applications, Springer-Verlag, Berlin Heidelberg, 2001.

Anteneodo, C. and Tsallis, C.: Two-dimensional turbulence in pureelectron plasma: A nonextensive thermostatistical description, J. Mol. Liq., 71, 255-267, 1997.

Arimitsu, T. and Arimitsu, N.: Analysis of fully developed turbulence in terms of Tsallis statistics, Phys. Rev. E, 61, 3237-3240, 2000.

Boghosian, B. M.: Thermodynamic Description of the Relaxation of Two-Dimensional Turbulence Using Tsallis Statistics, Phys. Rev. E, 53, 4754-4753, 1996.

Cheng, A. F. and Ruderman, M. A.: Particle Acceleration and Radio Emission Above Pulsar Polar Caps, Astrophys. J., 235, 576-586, 1980.

Collier, M. R., Hamilton, D. C., Gloeckler, G., Bochsler, P., and Sheldon, R. B.: Neon-20, Oxygen-16, and Helium-4 Densities, Temperatures, and Suprathermal Tails in the Solar Wind Determined with WIND/MASS, Geophys. Res. Lett., 23, 1191-1194, 1996.

Curtis, M. F.: The Theory of Neutron Stars Magnetospheres, University of Chicago Press, Chicago, 1991.

Fuchs, J., Adam, J. C., Amiranoff, F., Baton, S. D., Blanchot, N., Gallant, P., Gremillet, L., Héron, A., Kieffer, J. C., Laval, G., Malka, G., Miquel, J. L., Mora, P., Pépin, H., and Rousseaux, C.: Experimental Study of Laser Penetration in Overdense Plasmas at Relativistic Intensities. I: Hole Boring Through Preformed Plasmas Layers, Phys. Plasmas, 6, 2563-2568, 1999.

Hirotani, K., Iguchi, S., Kimura, M., and Wajima, K.: Pair Plasma Dominance in the Parsec-Scale Relativistic Jet of 3C 345, Astrophys. J., 545, 100-106, 2000.

Huang, X.-P. and Driscoll, C. F.: Relaxation of 2D Turbulence to a Metaequilibrium near the Minimum Enstrophy State, Phys. Rev. Lett., 72, 2187-2190, 1994.

Latora, V., Rapisarda, A., and Tsallis, C.: Non-Gaussian equilibrium in a long-range Hamiltonian system, Phys. Rev. E, 64, $056134,2001$.

Lavagno, A.: Relativistic Nonextensive Thermodynamics, Phys. Lett. A, 301, 13-18, 2002.

Leubner, M. P.: Fundamental Issues on Kappa-Distributions in Space Plasmas and Interplanetary Proton Distributions, Phys. Plasmas, 11, 1308-1316, 2004.

Lima, J. A. S., Silva Jr., R., and Santos, J.: Plasma Oscillations and Nonextensive Statistics, Phys. Rev. E, 61, 3260-3263, 2000.

Liu, J. M., Groot, J. S. D., Matte, J. P., Johnston, T. W., and Drake, R. P.: Measurements of Inverse Bremsstrahlung Absorption and non-Maxwellian electron velocity distributions, Phys. Rev. Lett., 72, 2717-2720, 1994.

Luo, Q., Melrose, D. B., and Fussell, D.: Wave Dispersion in Gyrotropic Relativistic Pulsar Plasmas, Phys. Rev. E, 66, 026405 , 2002.
Marsch, E., Mühlhäuser, K.-H., Schwenn, R., Rosenbauer, H., Pilipp, W., and Neubauer, F. M.: Solar Wind Protons: Threedimensional velocity distributions and derived plasma parameters measured between 0.3 and 1 AU, J. Geophys. Res., 87, 52 72, 1982.

Mauk, B. H., Krimigis, S. M., Keath, E. P., Cheng, A. F., Armstrong, T. P., Lanzerotti, L. J., Gloeckler, G., and Hamilton, D. C.: The Hot Plasma and Radiation Environment of the Uranian Magnetosphere, J. Geophys. Res., 92, 15 283-15 308, 1987.

Mourou, G. and Umstadter, D.: Development and Applications of Compact High-Intensity Lasers, Phys. Fluids B, 4, 2315-2325, 1992.

Naumova, N. M., Bulanov, S. V., Esirkepov, T., Farina, D., Nishihara, K., Pegoraro, F., Ruhl, H., and Sakharov, A. S.: Formation of Electromagnetic Postsolitons in Plasmas, Phys. Rev. Lett., 87, $185004,2001$.

Peng, C.-K., Mietus, J., Hausdorff, J. M., Havlin, S., Stanley, H. E., and Goldberger, A. L.: Long-Range Anticorrelations and NonGaussian Behavior of the Heartbeat, Phys. Rev. Lett., 70, $1343-$ 1346, 1993.

Prato, D. and Tsallis, C.: Nonextensive Foundation of Lévy Distributions, Phys. Rev. E, 60, 2398-2401, 1999.

Ruderman, M. A. and Sutherland, P. G.: Theory of Pulsars: Polar Gaps, Sparks, and Coherent Microwave Radiation, Astrophys. J., 196, 51-72, 1975.

Sattin, F.: Derivation of Tsallis statistics from dynamical equations for a granular gas, J. Phys. A, 36, 1583-1591, 2003.

Schlickeiser, R., Vainio, R., Böttcher, M., Lerche, I., Pohl, M., and Schuster, C.: Conversion of Relativistic Pair Energy into Radiation in the Jets of Active Galactic Nuclei, Astron. Astrophys., 393, 69-87, 2002.

Scudder, J. D., E. C. Sittler, J., and Bridge, H. S.: A Survey of the Plasma Electron Environment of Jupiter: A View From Voyager, J. Geophys. Res., 86, 8157-8179, 1981.

Silva Jr., R., Plastino, A. R., and Lima, J.: A Maxwellian Path to the $q$-Nonextensive Velocity Distribution Function, Phys. Lett. A, 249, 401-408, 1998.

Taruya, A. and aki Sakagami, M.: Long-Term Evolution of Stellar Self-Gravitating Systems Away from Thermal Equilibrium: Connection with Nonextensive Statistics, Phys. Rev. Lett., 90, 181 101, 2003.

Tsallis, C.: Possible Generalization of Boltzmann-Gibbs Statistics, J. Stat. Phys., 52, 479-487, 1988.

Tsallis, C. and de Souza, A. M. C.: Nonlinear Inverse Bremsstrahlung Absorption and Nonextensive Thermostatistics, Phys. Lett. A, 235, 444-446, 1997.

Wardle, J. F. C. and Homan, D. C.: The Nature of Jets: Evidence from Circular Polarization Observations, in: Particles and Fields in Radio Galaxies, ASP Conference Proceedings Vol. 250, edited by: Laing, R. A. and Blundell, K. M., Astronomical Society of the Pacific, San Francisco, astro-ph/0011515, 2001.

Wardle, J. F. C., Homan, D. C., Ojha, R., and Roberts, D. H.: Electron-Positron Jets Associated with the Quasar 3C279, Nature, 395, 457-461, 1998. 